\title{
Pharmacokinetic Parameters Time Point Reference
}

National Cancer Institute

\section{Source}

National Cancer Institute. Pharmacokinetic Parameters Time Point Reference. NCI

Thesaurus. Code C119922.

The point in time that acts as a fixed reference point to a pharmacokinetic parameter assessment. 